\title{
Cross-Sectional Area Increase at Phase Transition on Compression: An Unexpected Phenomenon Observed in an Amide Monolayer
}

\author{
G. Brezesinski, ${ }^{\dagger}$ C. Stefaniu, ${ }^{\dagger}$ D. Nandy,${ }^{\ddagger}$ S. Dutta Banik, ${ }^{\ddagger}$ N. Nandi, ${ }^{\ddagger}$ and D. Vollhardt $*, \dagger$ \\ Max Planck Institute of Colloids and Interfaces, D-14424 Potsdam/Golm, Germany, and Department of \\ Chemistry, University of Kalyani, Kalyani, Nadia, West Bengal, 741235 India
}

Received: May 13, 2010; Revised Manuscript Received: August 15, 2010

\begin{abstract}
At low temperatures $\left(T \leq 10{ }^{\circ} \mathrm{C}\right)$, the surface pressure-area $(\pi-A)$ isotherms of some amphiphilic amides reveal a striking second critical point indicating the existence of a second phase transition between two condensed phases. Studies of 3-hydroxy- $N$-tridecyl propanoic acid amide (HTPA) monolayers have shown that this phase transition between two condensed phases is accompanied by an abrupt change of important 2D lattice parameters. Thorough grazing incidence X-ray diffraction (GIXD) studies have revealed a new phenomenon. The cross-sectional area $\left(A_{0}\right)$ of the alkyl chain of HTPA jumps from a smaller value in the phase at the lower surface pressure to a larger value in the phase existing at higher pressure. The opposite behavior should be expected and is usually noted in the lattice structures of Langmuir monolayers. The phase diagram of the HTPA monolayers is constructed on the basis of the equilibrium $\pi-A$ isotherms. The fact that two condensed monolayer phases exist and the phase observed above the fluid/condensed transition at higher temperature has a structure similar to that observed at high pressure and low temperatures, as observed by the GIXD experiments, can be thermodynamically explained by the generalized equation of state for Langmuir monolayers for the existence of two condensed phases. The IRRA spectra did not show a substantial change in the band positions below and above the phase transition between the two condensed states, but differences in the dichroic ratio indicate changes in the hydrogen bonding system. The computational studies, using the $\mathrm{ab}$ initio level of theory for the headgroup and the molecular mechanical theory for the alkyl chain part, provide a reasonable explanation for the unexpected, novel finding that the cross-sectional area of the alkyl chains jumps to larger values at the phase transition on increasing pressure. It is shown that shortening of the hydrogen bond separation at higher pressure in the headgroup region obviously drives the increase in separation between the alkyl chains, in reasonable agreement with the GIXD results.
\end{abstract}

\section{Introduction}

The characteristic features of Langmuir monolayers are sensitively determined by the chemical structure of the amphiphiles. ${ }^{1}$ In recent years, interesting information has been obtained on the effect of small changes in the molecular structure of the amphiphile on the main characteristics of the monolayer; in particular, the surface pressure-area per molecule $(\pi-A)$ isotherms, ${ }^{2}$ the shape and texture of the condensed phase domains, ${ }^{3,4}$ and the two-dimensional lattice structure. ${ }^{5}$ Correspondingly, new knowledge about the influence of the most important functional groups has been acquired by specific modification of the chemical headgroup structure. ${ }^{6,7}$

Monolayer studies of model amphiphiles containing amide and amine groups has attracted attention because these groups are an integral part of the general structure of sphingolipids, ${ }^{8,9}$ which seem to play an important role in those membrane parts that allow the transport into the cells, and of naturally occurring $N$-acylethanolamines ${ }^{10-12}$ and their precursors, $N$-acylphosphatidylethanolamines, with respect to their metabolism and their interaction with other membrane lipids and proteins.

In previous papers, we studied the main monolayer characteristics of various tailored amphiphiles containing a headgroup

* Corresponding author. Phone: -49-331-567-9258. Fax: -49-331-5679202. E-mail: dieter.vollhardt@mpikg.mpg.de.

${ }^{\dagger}$ Max Planck Institute of Colloids and Interfaces.

¥ University of Kalyani. that consists of an amide group and one or two hydroxyl groups separated by one or more (two or three) methylene groups. ${ }^{13-23}$

General information about the thermodynamics and phase behavior of amphiphilic monolayers can be obtained from the surface pressure area $(\pi-A)$ isotherms at different temperatures. $^{24,25}$ The temperature region at which the $\pi-A$ isotherms show a plateau region is of particular interest. The plateau region after the main phase transition point is attributed to the transition from a low density fluid (gaseous, G; liquid expanded, LE) state to a condensed (liquid-condensed, LC) state. It is of special interest that, at low temperatures, the $\pi-A$ isotherms of some amphiphilic amides reveal a striking second critical point at $A$ $<A_{\mathrm{c}}$, indicating the existence of a second phase transition between two condensed phases. Studies of 3-hydroxy- $N$-tridecyl propanoic acid amide $\left(\mathrm{C}_{13} \mathrm{H}_{27}-\mathrm{NH}-\mathrm{CO}-\mathrm{C}_{2} \mathrm{H}_{4} \mathrm{OH}, \mathrm{HTPA}\right)$ monolayers have shown that this phase transition between two condensed phases is accompanied by an abrupt change of important 2D lattice parameters, such as molecular tilt angle and unit cell area $A_{x y}$, obtained by grazing incidence X-ray diffraction (GIXD). ${ }^{25,26}$ Despite the drastic changes in the lattice parameters both condensed phases had an oblique lattice.

These unusual properties were the reason to perform further careful studies of the phase behavior and lattice structure of 3 -hydroxy- $N$-tridecyl propanoic acid amide monolayers. Thorough GIXD studies have revealed a new phenomenon, to the best of our knowledge never observed before. The crosssectional area $\left(A_{0}\right)$ of the alkyl chain of HTPA jumps from a 
smaller value in the phase at lower surface pressure to a larger value in the phase existing at higher pressure. The opposite behavior should be expected and is usually noted in the lattice structures of Langmuir monolayers. The present work focuses on this new, interesting phenomenon.

\section{Experimental Section}

Materials. The amphiphile HTPA was synthesized as described previously in detail. ${ }^{26}$ At room temperature, $\beta$-propiolactone dissolved in anhydrous 1,4-dioxane was added to tridecylamine dissolved in anhydrous 1,4-dioxane. The mixture was heated to $80{ }^{\circ} \mathrm{C}$ for $5 \mathrm{~h}$. After removing the volatiles in vacuum, the obtained HTPA was recrystallized several times from dioxane/pentane (8:2) and acetone/pentane (8:2) mixtures. The chemical purity of $\geq 99 \%$ was checked by elemental analysis and high-performance liquid chromatography. The used spreading solvent was chloroform (p.a. grade, Baker, Holland). Ultrapure water with a specific resistance of $18.2 \mathrm{M} \Omega \cdot \mathrm{cm}$ used for the monolayer experiments was obtained from a Millipore desktop system.

Surface Pressure Measurements and Brewster Angle Microscopy. Equilibrium surface pressure $(\pi-A)$ isotherms recorded at a compression rate of $\leq 10 \AA^{2} /($ molecule $\cdot$ min) were measured in an experimental setup consisting of a self-made, computer-interfaced film balance-coupled with a Brewster angle microscope (BAM1+, NFT, Göttingen, Germany) ${ }^{26}$ Using the Wilhelmy method, the surface pressure was measured with a roughened glass plate with an accuracy of $\pm 0.1 \mathrm{mN} / \mathrm{m}$, and the area per molecule, with $\pm 0.5 \AA^{2}$ accuracy.

The lateral resolution of the BAM1+ is $\sim 4 \mu \mathrm{m}$. Simple imaging processing software was used to optimize the contrast. More detailed information on the BAM method is given elsewhere (see, e.g., refs 3 and 27, and the references therein).

X-ray Diffraction Measurements. ${ }^{28-31}$ Crystalline domains in the monolayer at the air/water interface were investigated using grazing incidence X-ray diffraction measurements at the BW1 beamline, HASYLAB (DESY, Hamburg, Germany). At BW1, a monochromatic X-ray beam $(\lambda=1.304 \AA)$ struck the water surface at a grazing incidence angle, $\alpha_{i}=0.85 \alpha_{c}\left(\alpha_{c}=\right.$ $0.13^{\circ}$ ), and illuminated roughly $100 \mathrm{~mm}^{2}$ of the monolayer surface (with $2 \mathrm{~mm}$ width and $50 \mathrm{~mm}$ length of the footprint area). During the course of an experiment through a slow lateral movement of the trough, the X-ray footprint moved across the monolayer to avoid sample damage by the strong X-ray beam. A linear position-sensitive detector (PSD, OEM-100-M, Braun, Garching, Germany) measured the diffracted signal and was rotated to scan the in-plane $Q_{x y}$ component values of the scattering vector. The vertical channels of the PSD measured the out-of-plane $Q_{z}$ component of the scattering vector between 0.0 and $0.85 \AA^{-1}$. The diffraction data consisted of Bragg peaks at diagnostic $Q_{x y}$ values. These peaks were obtained by summing the diffracted intensity at each in-plane $Q_{x y}$ value over a defined vertical angle or $Q_{z}$ window. The in-plane lattice repeat distances, $d$, of the ordered structures in the monolayer were calculated from the Bragg peak positions: $d=2 \pi / Q_{x y}$. To access the extent of the crystalline order in the monolayer, the in-plane coherence length, $L_{x y}$, was approximated from the full-width at half-maximum (fwhm) of the Bragg peaks using $L_{x y} \sim 0.9(2 \pi)$ / fwhm $\left(Q_{x y}\right)$. The diffracted intensity normal to the interface was integrated over the $Q_{x y}$ window containing the diffraction peak to calculate the corresponding Bragg rod. The thickness of the monolayer was estimated from the fwhm of the Bragg rod using $0.9(2 \pi) / \mathrm{fwhm}\left(Q_{z}\right)$.
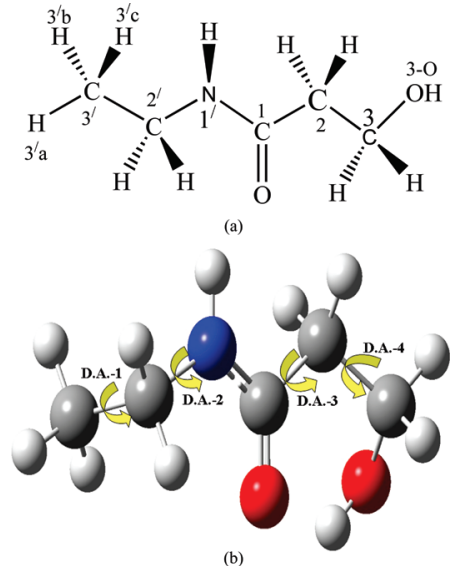

Figure 1. (a) Numbering scheme of the model structure of the HTPA headgroup. (b) Optimized geometry of the HTPA head using the HF/ $6-31 \mathrm{G}^{* *}$ level of theory. The dihedral angles [D.A.-1, $\mathrm{H}\left(3^{\prime} \mathrm{a}\right)-\mathrm{C}\left(3^{\prime}\right)-$ $\mathrm{C}\left(2^{\prime}\right)-\mathrm{N}\left(1^{\prime}\right)$; D.A.-2, $\mathrm{C}\left(3^{\prime}\right)-\mathrm{C}\left(2^{\prime}\right)-\mathrm{N}\left(1^{\prime}\right)-\mathrm{C}(1) ;$ D.A.-3, $\mathrm{N}\left(1^{\prime}\right)-$ $\mathrm{C}(1)-\mathrm{C}(2)-\mathrm{C}(3)$; D.A.-4, $\mathrm{C}(1)-\mathrm{C}(2)-\mathrm{C}(3)-\mathrm{O}(3-\mathrm{O})$ ] that are varied to generate the lowest energy conformer are shown by bold arrows.

IRRAS Measurements. ${ }^{32-36}$ In infrared reflection absorption spectroscopy (IRRAS), each infrared spectrum is a combination of two separate reflection measurements. The reported signal, in reflectance-absorbance (RA) units, is the ratio of the reflected light from two liquid surfaces: $\mathrm{RA}=-\log [$ (sample reflectivity)/ (reference reflectivity) $]=-\log (\mathrm{R} / \mathrm{R} 0)$. Measurements were performed using an IFS 66 FTIR spectrometer with a mercury-cadmium-telluride (MCT) detector attached to an external air/water reflection unit (XA-511, Bruker). A shuttling mechanism moved the small reference trough and the larger sample trough alternately into the IR beam path. The resolution and scanner speeds in all experiments were $8 \mathrm{~cm}^{-1}$ and $20 \mathrm{kHz}$. The incident IR beam was polarized with a KRS-5 wire grid polarizer. For s-polarized light, spectra were coadded over 200 scans and spectra with p-polarized light were coadded over 400 scans. The two different light polarizations provided information on molecular orientation with respect to the surface plane of the monolayer. A change in the intensity ratio of p-polarized to $\mathrm{s}$-polarized light ( $\mathrm{p} / \mathrm{s}$ ratio) for a vibrational band indicates a change in the average orientation of the vibration and, thus, of the molecule.

Theoretical Calculations. To generate the lowest-energy conformer of HTPA, the long alkyl chain (tridecyl group) was replaced by an ethyl group. The model structures are termed as HTPA headgroup (3-hydroxy- $N$-ethyl propanoic acid amide), as shown in Figure 1a. The model structure (HTPA headgroup) was optimized at the HF/6-31G** level of theory. The optimized structure, as shown in Figure 1b, was taken for further calculations relating to the monolayer features. The conformation of the lowest-energy structure is justified as follows. The following dihedral angles are varied in the headgroup region as $\mathrm{H}\left(3^{\prime} \mathrm{a}\right)-\mathrm{C}\left(3^{\prime}\right)-\mathrm{C}\left(2^{\prime}\right)-\mathrm{N}\left(1^{\prime}\right), \quad \mathrm{C}\left(3^{\prime}\right)-\mathrm{C}\left(2^{\prime}\right)-\mathrm{N}\left(1^{\prime}\right)-\mathrm{C}(1)$, $\mathrm{N}\left(1^{\prime}\right)-\mathrm{C}(1)-\mathrm{C}(2)-\mathrm{C}(3)$, and $\mathrm{C}(1)-\mathrm{C}(2)-\mathrm{C}(3)-\mathrm{O}(3-\mathrm{O})$, respectively. The numbering scheme is shown in Figure 1a.

Variation of $2 \pi$ with an increment of $30^{\circ}$ in each dihedral angle was made, and a total of 100 conformers were generated. The single-point energy of each conformer was calculated at the HF/6-31G** level of theory. The lowest-energy conformer obtained is the same conformer as obtained from optimization and confirms the lowest-energy structure at the level of theory considered. Subsequently, two-layered ONIOM (MO/MM) calculation was used for the energy minimization for the monomer structure for THPA with the alkyl chain part included 


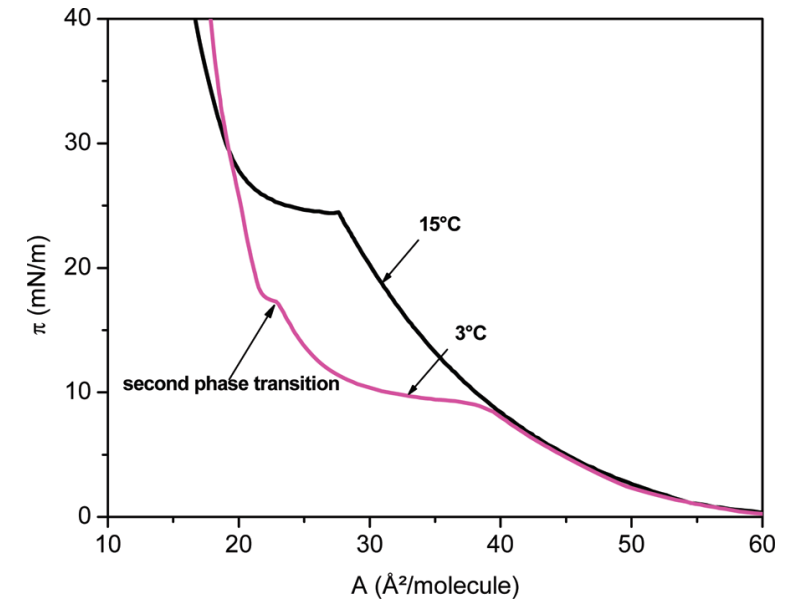

Figure 2. $\pi-A$ isotherms of HTPA monolayers spread on water at 3 and $15{ }^{\circ} \mathrm{C}$.

with the alkyl chain having the all-trans configuration. The ab initio level of theory (HF/6-31G**) was applied for the HTPA headgroup, and the molecular mechanical theory (UFF) was used for the alkyl chain part (undecyl group). The optimized structures of the monomer were used to form THPA dimers. Twenty-five different dimers were further optimized at same level of theory. The resulting structures have energy difference of 0 to $\sim 3 \mathrm{kcal} / \mathrm{mol}$ and differ in the hydrogen bonding pattern.

Similarly, tetramer structures were generated. A mode redundant optimization was carried out to find the hydrogen bond pattern at two different surface pressures. The separation between the carbon atoms joining the alkyl chain and the headgroup for each monomer in a tetrameric arrangement was kept fixed, whereas all other intra- and intermolecular variables were allowed for complete optimization. Whereas keeping the separation between the carbon atoms joining the alkyl chain and the headgroup for each monomer will mimic the molecular position at a given surface pressure and allow a given molecule the same area/molecule as present in that pressure, the flexible optimization of all other variables will allow the monomers to form a stable hydrogen bonded structure at that pressure. The tetramer energy for THPA at different pressures has been calculated at the same level of theory.

\section{Results}

Figure 2 shows two $\pi-A$ isotherms of the HTPA monolayers as characteristic examples for the low temperature range at 3 ${ }^{\circ} \mathrm{C}$ and for the high temperature range at $15^{\circ} \mathrm{C}$. The shapes of these isotherms are in complete agreement with those presented in a previous paper. ${ }^{26}$ Both isotherms show a sharp inflection point followed by an inclined plateau, indicating the first-order fluid/condensed phase transition. The $\pi-A$ isotherm at $15{ }^{\circ} \mathrm{C}$ corresponds to those of the most usual monolayers of amphiphiles. ${ }^{37}$ The lateral pressure of this main transition increases linearly with increasing temperature with a slope of $1.405 \mathrm{mN}$ / $\mathrm{m} \cdot \mathrm{K}^{-1}$, as concluded from several isotherms of ref. ${ }^{26}$

It is interesting to note that, at low temperatures, the $\pi-A$ isotherms at $3{ }^{\circ} \mathrm{C}$ (according to ref 26 at $<10{ }^{\circ} \mathrm{C}$ ) reveal a striking second weak inflection point at $A<A_{\mathrm{c}}$, indicating the existence of a second phase transition between two condensed phases. The area change after the second inflection point is very small compared with the main phase transition. The transition pressure of this second phase transition is nearly independent of temperature and amounts to $\sim 17.5 \mathrm{mN} / \mathrm{m}$. Therefore, the transition cannot be seen anymore at $\geq 10^{\circ} \mathrm{C}$ because then, the
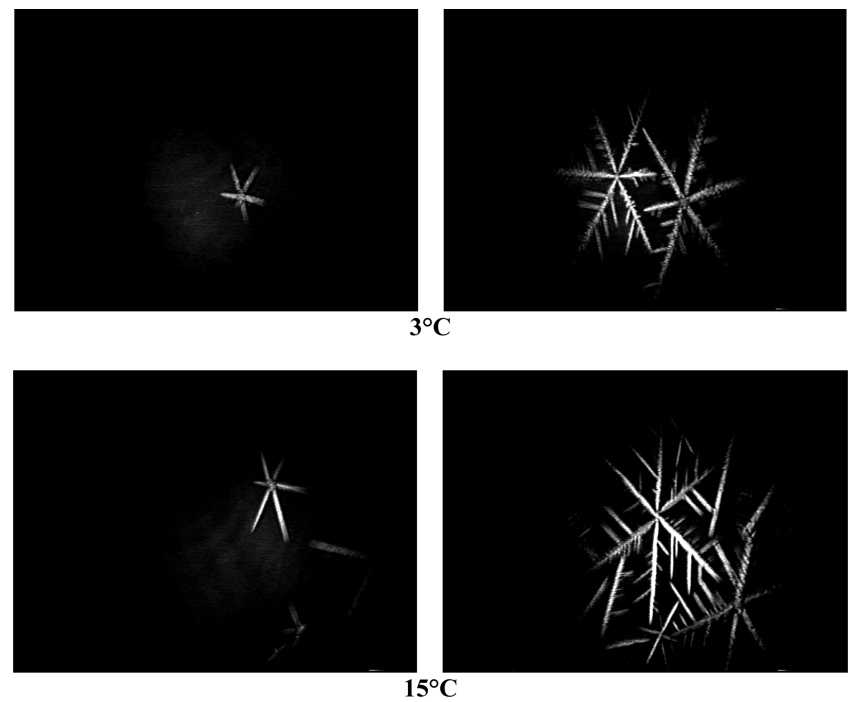

Figure 3. Domain growth within the fluid/condensed transition region of the $\pi-A$ isotherms of a HTPA monolayer spread on water.

main transition pressure is already higher than the expected pressure of this second phase transition. The formation of the condensed phase within the fluid/condensed phase transition region has been visualized by BAM experiments (Figure 3). At the beginning of the inclined plateau of the isotherms, domains with six straight main axes grow from a small round center in a regular distance of $\sim 60^{\circ}$ from each other independent of the temperature (Figure 3, left). The bright condensed phase domains are surrounded by the fluid phase of lower density and, thus, lower reflectivity. During the growth, the main axes develop numerous straight branches on both sides, clearly indicating the dendritic character of the domain shape (Figure 3 , right). The stability of the dendritic domains is remarkably high so that a rearrangement to a compact equilibrium shape could not be observed. This behavior is in agreement with experimental results of other amphiphiles containing an acid amide group.

GIXD gives information about the two-dimensional structure of monolayers on the $\AA$-scale. Therefore, it should be helpful to understand the nature of the second weak first-order transition seen in the isotherms at low temperatures and higher pressures. The diffraction studies were performed on water at two different temperatures $\left(3\right.$ and $15^{\circ} \mathrm{C}$ ) and at different lateral pressures. As expected from the isotherm measurements, condensed structures were not found at pressures below the first-order phase transition characterized by the extended plateau region.

Figure 4 shows selected contour plots of the corrected X-ray diffraction intensities as a function of the in-plane scattering vector component, $Q_{x y}$, and the out-of-plane scattering vector component, $Q_{z}$, obtained at different lateral pressures and temperatures.

The three Bragg peaks observed at all pressures and the two temperatures $\left(3\right.$ and $15^{\circ} \mathrm{C}$ ) investigated show that the structure of the monolayer remains oblique up to high lateral pressures. The alkyl chains are strongly tilted in a nonsymmetry direction. At $3{ }^{\circ} \mathrm{C}$, the observed structure at lateral pressures below the transition between the two condensed phases is quite similar to that of a rectangular unit cell with NN-tilted alkyl chains. At the transition pressures of $\sim 17.5 \mathrm{mN} / \mathrm{m}$, the structure changes to a more oblique lattice. As seen in Figure 4, similar contour plots, but with different peak and rod positions, have been found at higher temperature $\left(15^{\circ} \mathrm{C}\right)$. This means that the phase observed above the fluid/condensed (LE/LC) transition at higher 


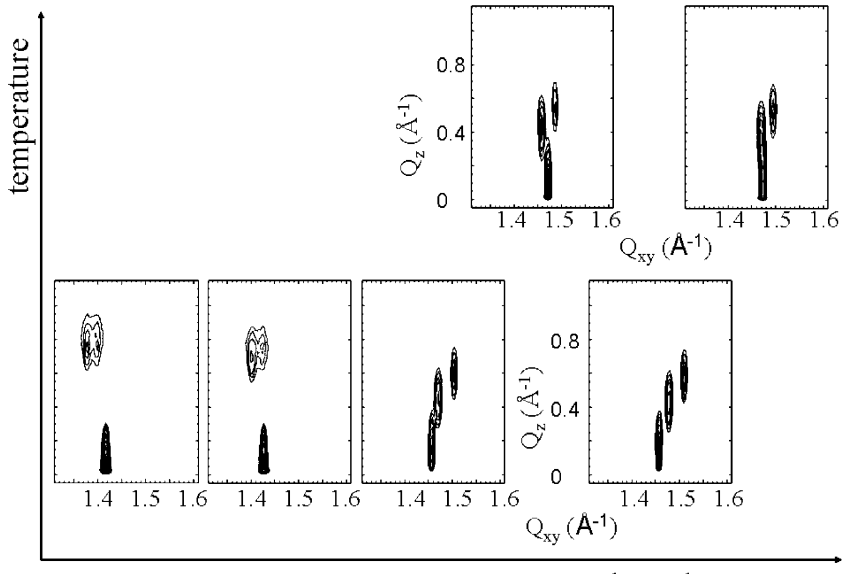

lateral pressure

Figure 4. GIXD contour plots of the corrected diffraction intensities as a function of the in-plane $Q_{x y}$ and out-of-plane $Q_{z}$ components of the scattering vectors for HTPA monolayers at different temperatures and lateral pressures. Top row: $15^{\circ} \mathrm{C}, 28$ and $35 \mathrm{mN} / \mathrm{m}$ (from left to right). Bottom row: $3{ }^{\circ} \mathrm{C} ; 12,18,24$, and $30 \mathrm{mN} / \mathrm{m}$ (from left to right).

TABLE 1: Lattice Parameters $a, b, c$ and $\alpha, \beta, \gamma$ of the Unit Cell; Lattice Distortion; Chain Tilt, $t$, from the Surface Normal; in-Plane Area, $A_{x y}$, per Chain; and Cross-Sectional Area, $A_{0}$, of HTPA Monolayers at $3{ }^{\circ} \mathrm{C}$ and Different Lateral Pressures $\pi$

\begin{tabular}{ccccccc}
\hline$\pi(\mathrm{mN} / \mathrm{m})$ & $\begin{array}{c}a, b, \\
c(\AA)\end{array}$ & $\begin{array}{c}\alpha, \beta, \\
\gamma\left({ }^{\circ}\right)\end{array}$ & distortion & $t\left(^{\circ}\right)$ & $A_{x y}\left(\AA^{2}\right)$ & $A_{0}\left(\AA^{2}\right)$ \\
\hline 12 & 5.123 & 121.4 & 0.0314 & 33.7 & 23.4 & 19.5 \\
& 5.202 & 119.9 & & & & \\
& 5.265 & 118.7 & & & & \\
17 & 5.059 & 121.2 & 0.0234 & 30.9 & 22.7 & 19.5 \\
& 5.149 & 119.4 & & & & \\
& 5.149 & 119.4 & & & & \\
20 & 4.866 & 121.2 & 0.0372 & 17.7 & 21.0 & 20.0 \\
& 4.899 & 120.6 & & & & \\
24 & 5.016 & 118.2 & & & & \\
& 4.845 & 121.4 & 0.0385 & 17.3 & 20.9 & 20.0 \\
& 4.895 & 120.4 & & & & \\
30 & 5.005 & 118.2 & & & & \\
& 4.818 & 121.6 & 0.0417 & 15.9 & 20.8 & 20.0 \\
& 4.888 & 120.3 & & & &
\end{tabular}

temperature has a structure similar to that observed at high pressure and low temperatures.

The lattice parameters of the HTPA monolayer measured at $3{ }^{\circ} \mathrm{C}$ are listed in Table 1 , and those measured at $15{ }^{\circ} \mathrm{C}$, in Table 2.

Calculating the lattice parameters from the X-ray data for this high pressure phase shows that the cross-sectional area, $A_{0}$, amounts to $20.2 \AA^{2}$ at $15{ }^{\circ} \mathrm{C}$ and $20.0 \AA^{2}$ at $3{ }^{\circ} \mathrm{C}$ (Figure 5).

These are typical values for a rotator phase, indicating free rotation of the alkyl chains. The slight increase in the crosssectional area is due to the increased temperature and is similar to changes observed with other amphiphiles. The tilt angle with respect to the surface normal, $t$, decreases only marginally with increasing pressure: $0.18 \% \mathrm{mN} / \mathrm{m}$ at $3{ }^{\circ} \mathrm{C}$ and $0.23^{\circ} / \mathrm{mN} / \mathrm{m}$ at $15{ }^{\circ} \mathrm{C}$. The transition pressure into a nontilted phase can be calculated using the following consideration. Since the condensed isotherms exhibit linear relations between pressure and molecular area and assuming a constant cross-sectional area in each phase, plotting of $1 / \cos (t)$ as a function of the lateral pressure allows the determination of the tilting phase transition pressure by extrapolation toward $1 /(\cos t)=1 .^{38}$ Figure 6 shows
TABLE 2: Lattice Parameters $a, b, c$ and $\alpha, \beta, \gamma$ of the Unit Cell; Lattice Distortion; Chain Tilt, $t$, from the Surface Normal; in-Plane Area, $A_{x y}$, per Chain; and Cross-Sectional Area, $A_{0}$, of HTPA Monolayers at $15{ }^{\circ} \mathrm{C}$ and Different Lateral Pressures, $\pi$

\begin{tabular}{ccccccc}
\hline \multirow{\pi}{\pi}{$(\mathrm{mN} / \mathrm{m})$} & $\begin{array}{c}a, b, \\
c(\AA)\end{array}$ & $\alpha, \beta$, & & & & \\
\hline 28 & 4.881 & 121.0 & 0.0227 & 16.2 & 21.0 & 20.2 \\
& 4.928 & 120.0 & & & & \\
& 4.978 & 119.0 & & & & \\
35 & 4.873 & 120.7 & 0.0219 & 14.8 & 20.8 & 20.1 \\
& 4.886 & 120.4 & & & & \\
4 & 4.959 & 118.9 & & & & \\
40 & 4.868 & 120.6 & 0.0225 & 13.4 & 20.8 & 20.2 \\
& 4.868 & 120.6 & & & & \\
& 4.950 & 118.9 & & & &
\end{tabular}

that the nontilted phase can be expected only at extremely high pressure values of around $70 \mathrm{mN} / \mathrm{m}$.

The low pressure phase at low temperatures is clearly different. On one hand, the tilt angle decreases more strongly with increasing pressure $\left(0.47^{\circ} / \mathrm{mN} \cdot \mathrm{m}^{-1}\right)$, and on the other hand, the cross-sectional area is much smaller $\left(19.5 \AA^{2}\right.$; see Figure 5 ), indicating a drastically reduced rotation of the alkyl chains.

This new phenomenon that the cross-sectional area increases on monolayer compression is very surprising. IRRAS experiments have been performed to obtain additional information for understanding such a behavior. IRRAS was mainly applied to check the existence of hydrogen bonds $(-\mathrm{NH} \cdots \mathrm{O}=\mathrm{C}-)$ in the monolayer. It is well-known that $\mathrm{H}$-bonds shift the bands of

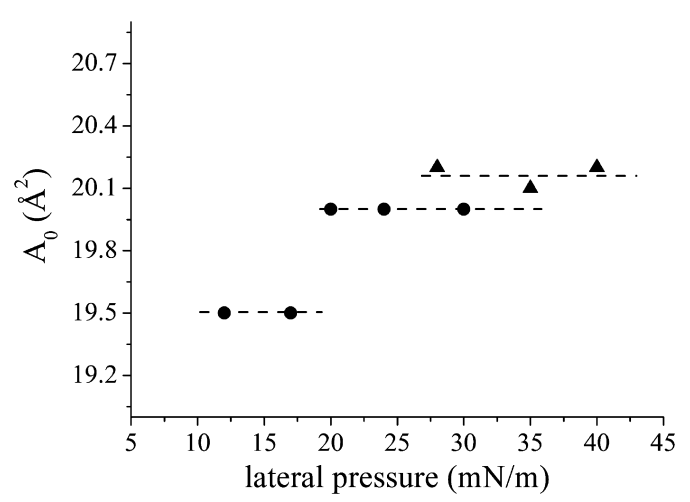

Figure 5. GIXD results of the cross-sectional area of alkyl chain $A_{0}$ in dependence on the lateral pressure $\pi$ at $3{ }^{\circ} \mathrm{C}(\bullet)$ and at $15{ }^{\circ} \mathrm{C}(\mathbf{\Delta})$.

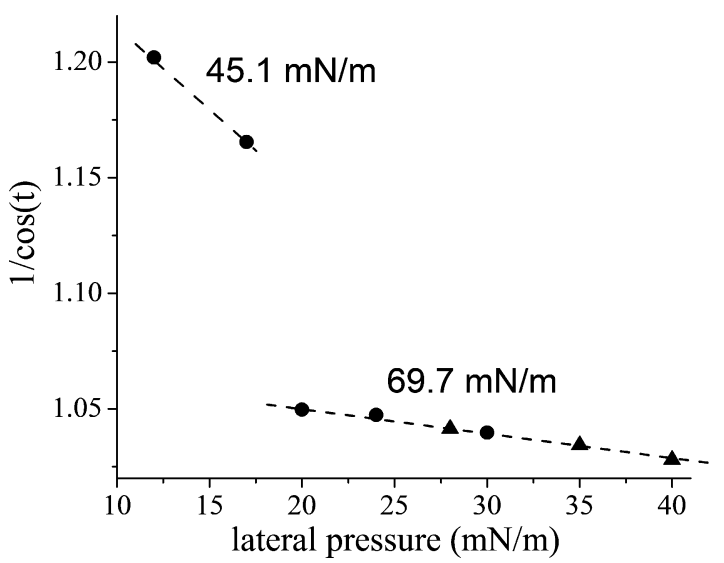

Figure 6. $1 /(\cos t)$ as function of the lateral pressure at $3{ }^{\circ} \mathrm{C}$. The linear extrapolation toward zero tilt angle $(1 /(\cos t)=1)$ yields the pressure of the tilting transition. 


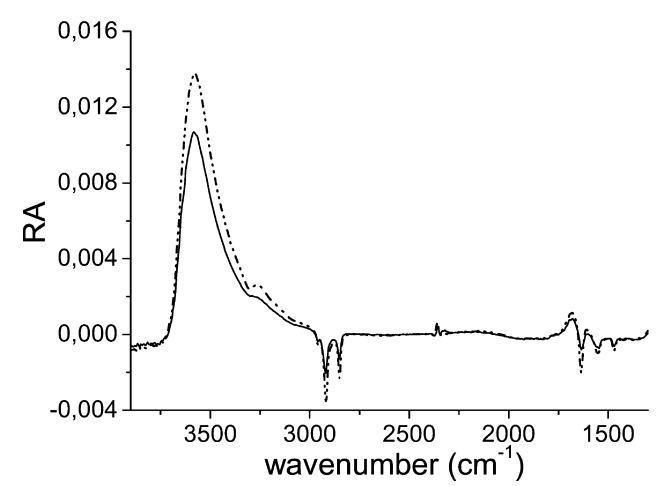

Figure 7. IRRA spectra of a HTPA monolayer at 14 (solid line) and 25 (dotted-dashed line) $\mathrm{mN} / \mathrm{m}$ measured at $3{ }^{\circ} \mathrm{C}$ with s-polarized light at an angle of incidence of $45^{\circ}$.

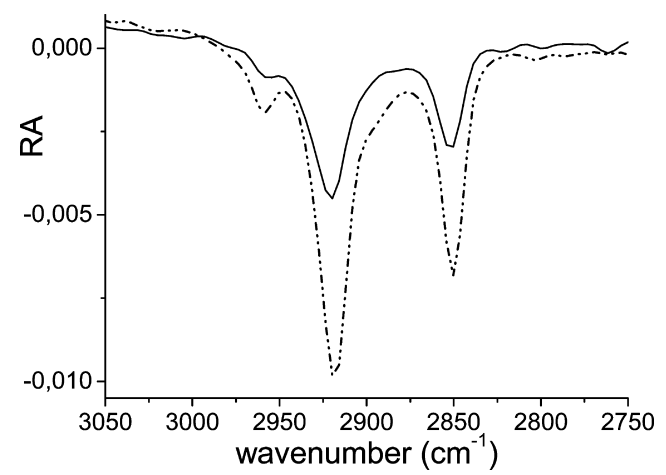

Figure 8. The $\mathrm{CH}$ stretching vibration region of IRRA spectra of HTPA monolayer at $14 \mathrm{mN} / \mathrm{m}$ (solid line) and at $25 \mathrm{mN} / \mathrm{m}$ (dotted-dashed line) measured at $3{ }^{\circ} \mathrm{C}$ with p-polarized light at an angle of incidence of $45^{\circ}$.

the stretching vibrations $v(\mathrm{NH})$ and $v(\mathrm{C}=\mathrm{O})$ to lower wavenumbers and that of the deformation peak $\delta(\mathrm{NH})$ in the opposite direction. $^{39}$

For secondary amides, the $v(\mathrm{NH})$ peak has different locations for trans and cis conformations of the amide group. H-bonded trans $\mathrm{NH}$ groups, with $\mathrm{N}-\mathrm{H}$ and $\mathrm{C}=\mathrm{O}$ bonds pointing in opposite directions, exhibit the $v(\mathrm{NH})$ band at $3320-3270 \mathrm{~cm}^{-1}$. For cis $\mathrm{NH}$ groups, with $\mathrm{N}-\mathrm{H}$ and $\mathrm{C}=\mathrm{O}$ bonds pointing in the same direction, this band appears at $3180-3140 \mathrm{~cm}^{-1}$. The trans polar heads are typical for the solid state of open-chain secondary amides $\mathrm{R}_{1}-\mathrm{NHCO}-\mathrm{R}_{2}$, where the $-\mathrm{NH} \cdots \mathrm{O}=\mathrm{C}-$ bonds form a lateral intermolecular network. Cyclic secondary amides usually have cis polar heads, which form $\mathrm{H}$-bonded dimers. The $\mathrm{R}_{1}-\mathrm{NH}-\mathrm{CO}-\mathrm{R}_{2}$ amides exhibit an intensive $v(\mathrm{C}=\mathrm{O})$ band (amide $\mathrm{I}$ ), whose position is very sensitive to molecular association. In dilute inert solutions (no hydrogen bonds), this peak appears at $1700-1670 \mathrm{~cm}^{-1}$. The $-\mathrm{NH} \cdot \cdots$ $\mathrm{O}=\mathrm{C}-$ bonds in the solid state shift this peak to $1680-1630$ $\mathrm{cm}^{-1}$. An amide II band that is specific for the noncyclic secondary amides appears in the range $1540-1510 \mathrm{~cm}^{-1}$ for dilute inert solutions and at $\sim 1550 \mathrm{~cm}^{-1}$ for the solid state. Its nature is complex $v(\mathrm{CN})+\delta(\mathrm{NH})$, but the red shift caused by the breaking of the hydrogen bonds in dilute inert solutions suggests that its deformation component $\delta(\mathrm{NH})$ plays the main role. $^{40}$

Figure 7 shows the IRRA spectrum of the HTPA monolayer at two different lateral pressures below and above the observed transition between the two condensed phases. The most noticeable feature in the spectra is the band at $3580 \mathrm{~cm}^{-1}$ that arises from the $\mathrm{OH}$ stretch of water and is a characteristic feature of IRRA spectra. The water $\mathrm{OH}$ stretching vibration present in the

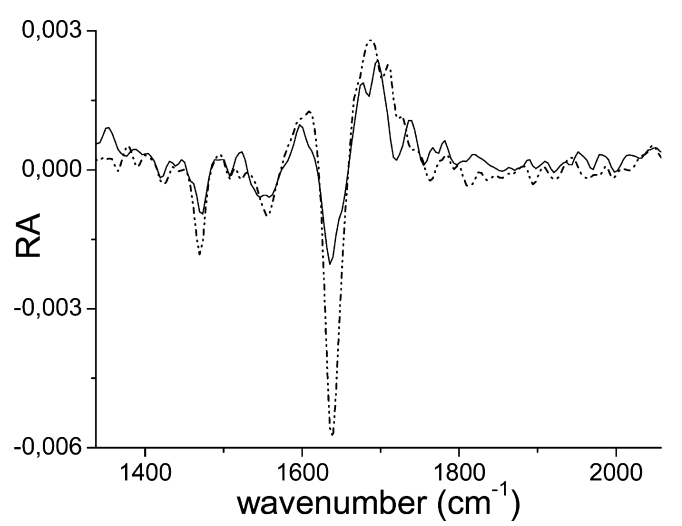

Figure 9. The amide I and II regions of IRRA spectra of HTPA monolayer at 14 (solid line) and at $25 \mathrm{mN} / \mathrm{m}$ (dotted-dashed line) measured at $3{ }^{\circ} \mathrm{C}$ with p-polarized light at an angle of incidence of $45^{\circ}$.

reference signal $\left(R_{0}\right)$ is reduced in the reflectivity signal from the monolayer-covered surface $(R)$ because the HTPA layer replaces a water layer and masks partially the $\mathrm{OH}$ stretching vibration. The result is a strong positive band at $3580 \mathrm{~cm}^{-1}$ that is related to the monolayer's effective thickness. The increase in RA intensity of this band at higher pressure is attributed to the effectively thicker HTPA layer at the surface because of the smaller tilt observed in the GIXD experiments.

The $\mathrm{CH}$ stretching vibration region $\left(2840-2975 \mathrm{~cm}^{-1}\right)$ is characterized by two prominent bands (symmetric and asymmetric $\mathrm{CH}_{2}$ stretching vibrations) and one much weaker band (asymmetric $\mathrm{CH}_{3}$ stretching vibration). The position of the $\mathrm{CH}_{2}$ stretching bands shifts slightly to lower wavenumber on compression from 14 to $25 \mathrm{mN} / \mathrm{m}$ (from 2852.5 to $2850.5 \mathrm{~cm}^{-1}$ for the symmetric and from 2920.8 to $2918.7 \mathrm{~cm}^{-1}$ for the asymmetric stretching vibration). The $\mathrm{CH}_{3}$ asymmetric stretching can be better seen at higher pressure and is located at 2958.4 $\mathrm{cm}^{-1}$. The observed band positions clearly indicate a wellpacked condensed monolayer with all-trans conformation of the alkyl chains. The shift of the $\mathrm{CH}_{2}$ stretching bands shows that the number of trans conformations in the alkyl chains increases on compression, what corresponds to a decrease of defects in the monolayer. The intensity of the bands measured with $\mathrm{p}$ and s-polarized light increases with increasing pressure, indicating a higher packing density in the area of the IR beam. The dichroic ratio $\left(A_{\mathrm{p}} / A_{\mathrm{s}}\right)$ also increases on compression, in accordance with the observed change of the tilt angle.

The HTPA molecules in $\mathrm{CCl}_{4}$ solution show a single $v(\mathrm{NH})$ band at $3462 \mathrm{~cm}^{-1}$, typical for the free $\mathrm{NH}$ stretching of trans polar heads of secondary amides. In contrast, the IRRA spectrum of the HTPA monolayer reveals a single band but at $3307 \mathrm{~cm}^{-1}$, indicating that the NH groups of the HTPA monolayer are trans oriented and $\mathrm{H}$-bonded. The absence of the free $v(\mathrm{NH})$ peak at $3462 \mathrm{~cm}^{-1}$ proves that all $\mathrm{NH}$ groups in the condensed monolayer phase are involved in H-bonds. ${ }^{34}$

The existence of an extended hydrogen bonding network is supported by the positions of the amide I $\left(1637 \mathrm{~cm}^{-1}\right)$ and amide II $\left(1552 \mathrm{~cm}^{-1}\right.$ ) bands (Figure 9). In dilute $\mathrm{CCl}_{4}$ solution (not shown), the amide I peak is located at $1706 \mathrm{~cm}^{-1}$, and the amide II peak, at $1508 \mathrm{~cm}^{-1}$. The $\mathrm{CH}_{2}$ deformation band appears as a single peak at $1470 \mathrm{~cm}^{-1}$ (Figure 9). Packing in an orthorhombic unit cell would lead to a splitting of this band. This is not observed, in accordance with the above GIXD data.

Summarizing, the comparison of the IRRA spectra below and above the phase transition between the two condensed states has shown that no substantial change in the band positions can 


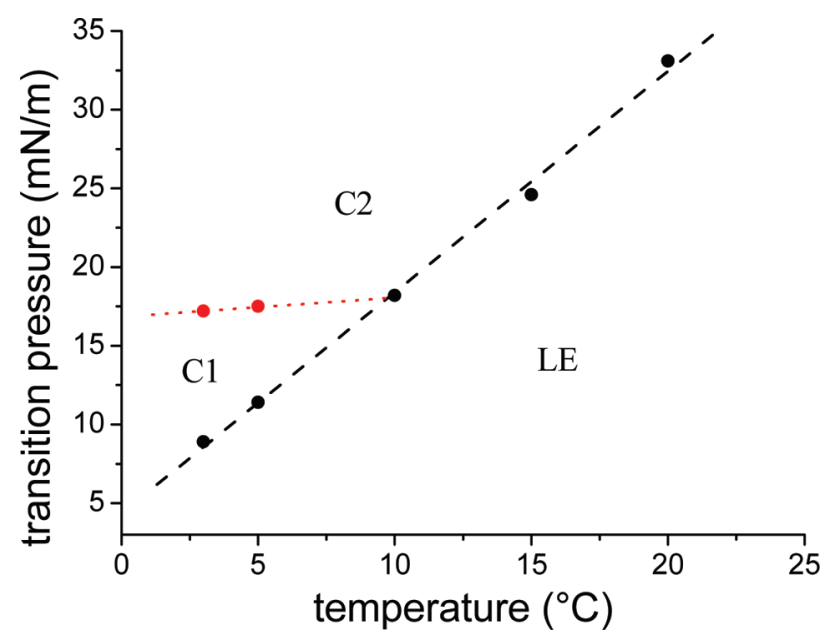

Figure 10. Phase diagram of HTPA monolayer: LE, fluid phase (liquidexpanded, gaseous); $\mathrm{C} 1$, low-temperature condensed phase; $\mathrm{C} 2$, hightemperature condensed phase.

be observed. However, a change in the orientation of the dipole transition moment during the phase transition is indicated by differences in the dichroic ratio. The dichroic ratio (i.e., the intensities of amide I band at $1637 \mathrm{~cm}^{-1}$ using $\mathrm{p}$ and s polarized light) is around 4 at lateral pressures below the phase transition and around 3 above the transition pressure.

\section{Discussion}

The HTPA monolayers show two types of $\pi-A$ isotherms: (i) at low temperatures $\left(T<10^{\circ} \mathrm{C}\right)$, with two first order phase transition; and (ii) at higher temperatures $\left(T \geq 10^{\circ} \mathrm{C}\right)$, only one first order phase transition (Figure 1 and Figure 3 of ref 26). This can be demonstrated by a simple transition pressuretemperature phase diagram that shows the existence regions of the disordered LE and the two condensed phases, $\mathrm{C} 1$ and $\mathrm{C} 2$ (Figure 10).

The thermodynamic explanation for this behavior can be concluded from the generalized equation of state for Langmuir monolayers for the case that two condensed phases can exist in the monolayer. ${ }^{23}$ For the two condensed phases $\mathrm{C} 1$ and $\mathrm{C} 2$ with the common fluid (LE, gaseous) branch of the isotherm, the shape of their isotherms can be determined (Figure 11). All necessary model parameters for the equation of state under formation of the two condensed phases are either calculated from the gaseous monolayer branch of the isotherm or are evaluated from the results of the GIXD experiment. Considering that the stability condition determines the behavior of a thermodynamic system, the relevant criterion for spread or adsorbed monolayers is $\mathrm{d} \pi / \mathrm{dA} \leq 0 .{ }^{41}$ That means that the dependence of the surface pressure on the area per molecule must have a minimum slope to be thermodynamically stable. The thermodynamically stable course of the HTPA isotherm of $3{ }^{\circ} \mathrm{C}$ is shown by the arrows in Figure 11. According to the above criterion, the system follows the lowest line of the two routes possible in points $\mathrm{A}$ and $\mathrm{C}$. That is, the route proceeds in point $\mathrm{A}$ along curve 1 across the coexistence region of the condensed phase 1 , whereas it proceeds in point $\mathrm{C}$ along line 2 , corresponding to the condensed state 2 , which possesses the lower slope. According to the temperature dependence of the HTPA isotherms, point A approaches point B, and at higher temperatures $\left(T \geq 17.5^{\circ} \mathrm{C}\right)$ only one first order phase transition exists. Hence, under consideration of the thermodynamic stability criterion, the second condensed phase cannot be realized, and only one condensed phase is formed. Correspond-

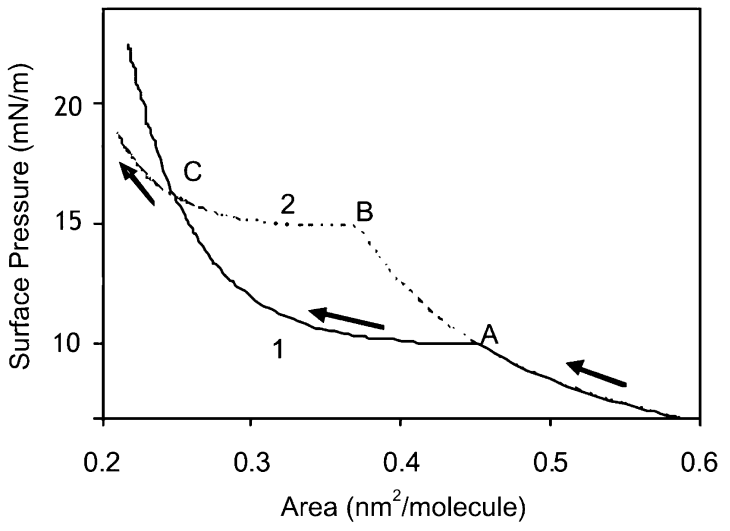

Figure 11. Theoretical $\pi-A$ isotherms 1 and 2 for the HTPA monolayer, calculated from the equations of state with the corresponding parameters: $\omega$, the partial molecular area for monomers (or the limiting area of a molecule in the gaseous state); $A_{\mathrm{c}}$, the molecular area that corresponds to the onset of the phase transition; $\eta$, a relative twodimensional compressibility; $\varepsilon_{0}$, the relative jump of the area per molecule; $m$, the aggregation number for small aggregates; and $\pi_{\mathrm{coh}}$, the cohesion pressure, according to ref 23 .

ingly, the phase observed above the fluid/condensed transition at higher temperature has a similar structure as that observed at high pressure and low temperatures in agreement with the GIXD results (see Figure 5).

However, the new interesting phenomenon presented in Figure 5 that the cross sectional area $\left(A_{0}\right)$ of the alkyl chain of HTPA jumps from a smaller value in the phase at the lower surface pressure to a higher value in the phase existing at higher pressure should be discussed in more detail. The phase observed at high pressure and low temperatures has a structure similar to that observed above the LE/LC transition at higher temperature. The cross-sectional area for a rotator phase indicates free rotation of the alkyl chains, and its slight increase with increased temperature is similar to changes observed with other amphiphiles. On the other hand, the cross-sectional area of the low pressure phase at low temperatures is much smaller $\left(A_{0}=19.5\right.$ $\AA^{2}$; see Figure 5), indicating a drastically reduced rotation of the alkyl chains. The opposite behavior should be expected. However, the tilt angle decreases strongly with increasing pressure, as expected. To check the role of hydrogen bonds $(-\mathrm{NH} \cdots \mathrm{O}=\mathrm{C}-)$ for this behavior, IRRAS experiments have been performed.

The comparison of the IRRA spectra below and above the phase transition between the two condensed states did not show substantial changes in the band positions; merely, a change in the orientation of the dipole transition moment during the phase transition indicated by differences in the dichroic ratio suggests changes in the hydrogen bonding system.

In the following, a detailed theoretical analysis of the hydrogen bonded network of the HTPA monolayer at different surface pressures has been performed to contribute to the understanding of the observed new phenomenon that the crosssectional area of the alkyl chain of HTPA jumps from a smaller value at the lower surface pressure to a larger value in the higher pressure phase. According to the above calculation procedure using the ab initio level of theory (HF/6-31G**) for the headgroup and the molecular mechanical theory for the alkyl chain part, it was possible to determine the molecular position in a tetrameric arrangement at the two surface pressures of 12 and $30 \mathrm{mN} / \mathrm{m}$.

The tetramer energy for HTPA is calculated at the same level of theory at the lateral pressures of 12 and at $30 \mathrm{mN} / \mathrm{m}$, 

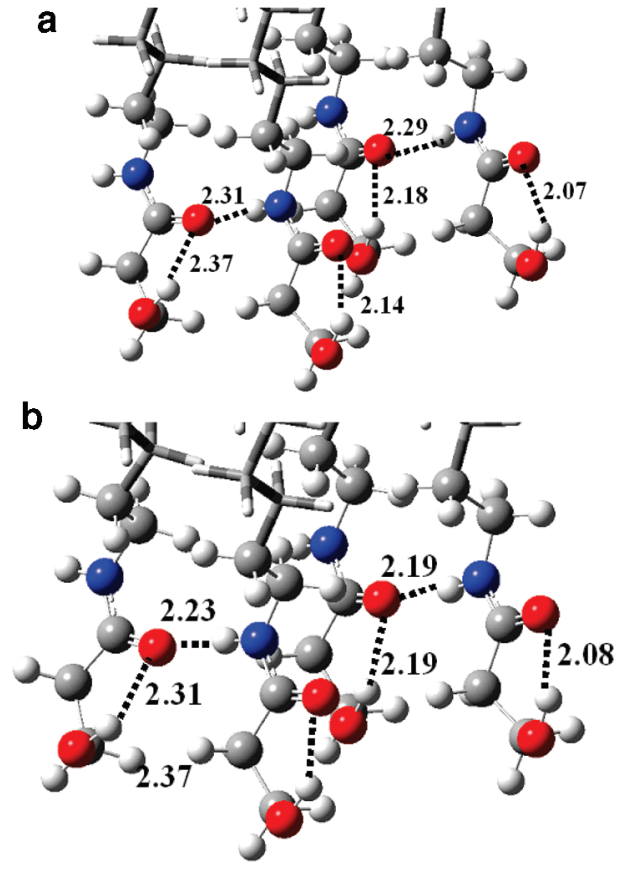

Figure 12. (a) Hydrogen bonding pattern for tetramer of HTPA molecule at $\pi=12 \mathrm{mN} / \mathrm{m}$. (b) Hydrogen bonding pattern for tetramer of HTPA molecule at $\pi=30 \mathrm{mN} / \mathrm{m}$.

respectively. The difference in energies of the tetramer at 12 and $30 \mathrm{mN} / \mathrm{m}$ is $-0.002359 \mathrm{au}$, which is equivalent to -1.48 $\mathrm{kcal} / \mathrm{mol}(1 \mathrm{au}=627.51 \mathrm{kcal} / \mathrm{mol})$. The HTPA tetramer is thus stabilized by $1.48 \mathrm{kcal} / \mathrm{mol}$ at lateral pressure of $30 \mathrm{mN} / \mathrm{m}$ relative to the state at $12 \mathrm{mN} / \mathrm{m}$.

Both $-\mathrm{C}=\mathrm{O} / \mathrm{H}-\mathrm{N}-$ intermolecular hydrogen bonds and $-\mathrm{C}=\mathrm{O} / \mathrm{H}-\mathrm{O}-$ intramolecular hydrogen bonds are noted in the tetramer structure. The hydrogen bonding pattern is shown in Figure $12 \mathrm{a}, \mathrm{b}$ for $\pi=12$ and $30 \mathrm{mN} / \mathrm{m}$, respectively. On an average, a decrement of the length of the hydrogen bonds are noted on going from $\pi=12$ to $30 \mathrm{mN} / \mathrm{m}$. This indicates a stronger hydrogen bond structure at higher pressure.

Keeping the separation between the carbon atoms joining the alkyl chain and the headgroup for each monomer the tetrameric arrangement mimics the molecular position at a given surface pressure. The flexible optimization of all other variables allows the monomers to form the stable hydrogen bonded structure at the respective lateral pressure, and consequently, a given molecule occupies the same area/molecule as that present in that pressure. The tetrameric arrangement of the mode redundant optimized THPA structure is shown in Figure 13a, b for $\pi=12$ and $30 \mathrm{mN} / \mathrm{m}$, respectively. Interestingly, the spacing between the alkyl chains along the lattice arms of the tetramer are 4.365 and $4.155 \AA$ at $\pi=12 \mathrm{mN} / \mathrm{m}$ and are 4.425 and $4.17 \AA$ at $\pi=$ $30 \mathrm{mN} / \mathrm{m}$. This conclusively shows that the spacing between the alkyl chains increases with increasing surface pressure. The shortening of the hydrogen bond separation at higher pressure in the headgroup region obviously drives the increase in separation between the alkyl chains, in agreement with the GIXD results that the cross-sectional area of alkyl chains, $A_{0}$, increases with the lateral pressure. Thus, this computational study provides a reasonable explanation for the unexpected novel finding that the cross-sectional area of the alkyl chains jumps to larger values during the phase transition at higher pressure. In comparison with a small number of molecules, such as the tetramer considered in the calculations here, this effect is expected to be more pronounced in an extended monolayer in which the HTPA amphiphiles form a continuous structure and will exhibit hydrogen bond cooperativity.
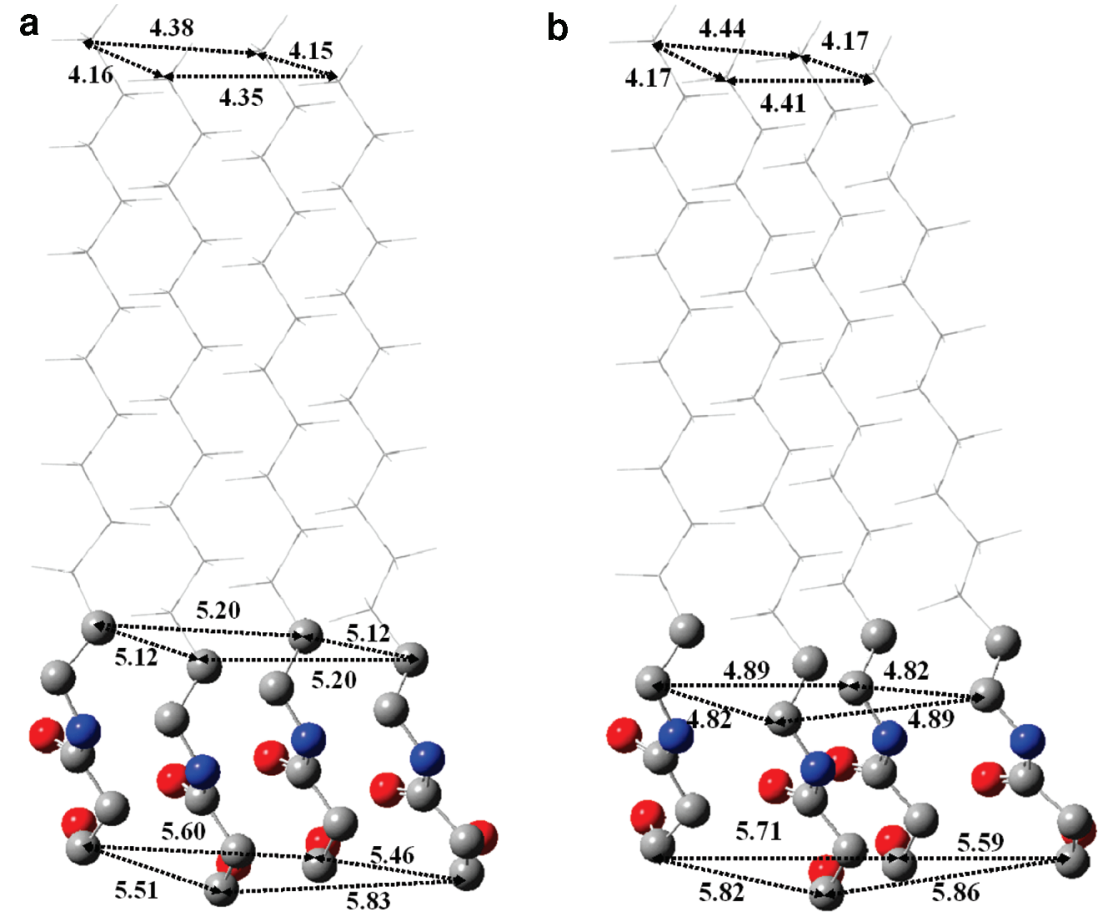

Figure 13. (a) Mode redundant optimized structure of tetramer of HTPA at $\pi=12 \mathrm{mN} / \mathrm{m}$ using two-level ONIOM (HF/6-31G**: UFF) method. The head part of HTPA (termed as THPA headgroup) (see text for details) is shown in the ball-and-bond type model, and the long alkyl chain is shown in the stick model. The hydrogen atoms are not shown. Selected parameters are shown in this figure. (b) Mode redundant optimized structure of tetramer of HTPA at $\pi=30 \mathrm{mN} / \mathrm{m}$ using two-level ONIOM (HF/6-31G**: UFF) method. The head part of HTPA (termed as HTPA headgroup) (see text for details) is shown in the ball-and-bond type model, and the long alkyl chain is shown in the stick model. The hydrogen atoms are not shown. Selected parameters are shown in this figure. 


\section{Conclusions}

Some amphiphilic amides show two types of $\pi-A$ isotherms: (i) at low temperatures $\left(T<10^{\circ} \mathrm{C}\right)$ with two first order phase transitions and (ii) at higher temperatures $\left(T \geq 10{ }^{\circ} \mathrm{C}\right)$ with only one first order phase transition. Studies of 3-hydroxy- $N$ tridecyl propanoic acid amide (HTPA) monolayers have demonstrated that this phase transition between two condensed phases is accompanied by an abrupt change of important 2D lattice parameters, such as the molecular tilt angle and unit cell area $A_{x y}$. Despite the drastic changes in the lattice parameters, both condensed phases have an oblique lattice.

Thorough grazing incidence X-ray diffraction studies have revealed a new phenomenon. The cross-sectional area of the alkyl chain of HTPA jumps from a smaller value in the phase at the lower surface pressure to a larger value in the phase existing at higher pressure. The opposite behavior should be expected and is usually observed in the lattice structures of other amphiphilic monolayers.

The phase diagram of the HTPA monolayers is constructed on the basis of the equilibrium $\pi-A$ isotherms. The fact that two condensed monolayer phases exist at low temperatures and the phase observed above the fluid/condensed (LE/LC) transition at higher temperature has a structure similar to that observed at high pressure and low temperatures, as observed by the GIXD experiments, can be thermodynamically explained by the generalized equation of state for Langmuir monolayers ${ }^{23}$ for the existence of two condensed phases.

The IRRA spectra did not show a substantial change in the band positions below and above the phase transition between the two condensed states, but differences in the dichroic ratio indicate changes in the hydrogen bonding system. The computational studies, using the $a b$ initio level of theory for the headgroup and the molecular mechanical theory for the alkyl chain part provide a reasonable explanation for the unexpected novel finding that the cross-sectional area of the alkyl chains jumps to larger values during the phase transition at higher pressure. It is shown that shortening of the hydrogen bond separation at higher pressure in the headgroup region obviously drives the increase in separation between the alkyl chains, in reasonable agreement with the GIXD results.

\section{References and Notes}

(1) McConnell, H. M. Annu. Rev. Phys. Chem. 1991, 42, 171-195. 7670 .

(2) Vollhardt, D.; Siegel, S.; Cadenhead, D. A. Langmuir 2004, 20 ,

(3) Vollhardt, D. Adv. Colloid Interface Sci. 1996, 64, 143-171.

(4) Vollhardt, D. In Encyclopedia of Surface and Colloid Science, 2nd ed.; Somasundaran, P., Ed.; Taylor \& Francis: New York, 2006: Vol. 5, pp $4104-4118$

(5) Kaganer, V. M.; Möhwald, H.; Dutta, P. Rev. Mod. Phys. 1999, $71,779-819$.

(6) Weidemann, G.; Vollhardt, D. Biophys. J. 1996, 70, 2758-2766.

(7) Thirumoorthy, K.; Nandi, N.; Vollhardt, D. J. Phys. Chem. B 2005, 109, 10820-10829.
(8) Nelson, D. L.; Cox, M. M.; Lehninger, A. L. Principles of Biochemistry, 4th ed.; W. H. Freeman \& Co.: New York, 2004; p 353.

(9) Mombelli, E.; Morris, R.; Taylor, W.; Fraternali, F. Biophys. J. 2003, 84, 1507-1517.

(10) Schmid, H. H. O.; Schmid, P. C.; Natarajan, V. Prog. Lipid Res. 1990, 29, 1-43.

(11) Hansen, H. S.; Moesgaard, B.; Hansen, H. H.; Petersen, G. Chem. Phys. Lipids. 2000, 108, 135-150.

(12) Kamlekar, R. K.; Satyanarayana, S.; Marsh, D.; Swamy, M. J. Biophys. J. 2007, 92, 3968-3977.

(13) Melzer, V.; Vollhardt, D. Phys. Rev. Lett. 1996, 76, 3770-3773.

(14) Vollhardt, D.; Melzer, V. J. Phys. Chem. B 1997, 101, 3370-3375.

(15) Melzer, V.; Weidemann, G.; Volhardt, D.; Brezesinski, G.; Wagner,

R.; Struth, B.; Möhwald, H. J. Phys. Chem. B 1997, 101, 4752-4758.

(16) Melzer, V.; Weidemann, G.; Vollhardt, D.; Brezesinski, G.; Wagner, R.; Struth, B.; Möhwald, H. Supramol. Sci. 1997, 4, 391-397.

(17) Melzer, V.; Vollhardt, D. Prog. Colloid Polym. Sci. 1997, 105, 130-137.

(18) Melzer, V.; Vollhardt, D.; Brezesinski, G.; Möhwald, H. J. Phys. Chem B 1998, 102, 591-597.

(19) Melzer, V.; Vollhardt, D.; Weidemann, G.; Brezesinski, G.; Wagner, R.; Möhwald, H. Phys. Rev. E 1998, 57, 901-907.

(20) Vollhardt, D.; Melzer, V.; Fainermann, V. B. Thin Solid Films 1998, 327-329, 842-845.

(21) Melzer, V.; Vollhardt, D.; Brezesinski, G.; Möhwald, H. Thin Solid Films 1998, 327-329, 857-860.

(22) Melzer, V.; Weidemann, G.; Wagner, R.; Vollhardt, D.; DeWolf, C.; Brezesinski, G.; Möhwald, H. Chem. Eng. Technol. 1998, 21, 44-48.

(23) Fainerman, V. B.; Vollhardt, D. J. Phys. Chem. B 2003, 107, 30983100.

(24) Fainerman, V. B.; Vollhardt, D. J. Phys. Chem. B 1999, 103, 145150.

(25) Fainerman, V. B.; Vollhardt, D. J. Phys. Chem. B 2009, 113, 63116313.

(26) Vollhardt, D.; Wagner, R. J. Phys. Chem. B 2006, 110, 1488114889.

(27) Meunier, J. Colloids Surf. A 2000, 171, 33

(28) Als-Nielsen, J.; Jaquemain, D.; Kjaer, K.; Lahav, M.; Levellier, F.; Leiserowitz, L. Phys. Rep. 1994, 246, 251-313.

(29) Kjaer, K. Physica B 1994, 198, 100-109.

(30) Rietz, R.; Rettig, W.; Brezesinski, G.; Bouwman, W. G.; Kjaer, K.; Möhwald, H. Thin Solid Films 1996, 285, 211-215.

(31) Lepere, M.; Chevallard, C.; Brezesinski, G.; Goldmann, M.; Guenoun, P. Angew. Chem., Int. Ed. 2009, 48, 5005-5009.

(32) Mendelsohn, R.; Brauner, J. W.; Gericke, A. Annu. Rev. Phys. Chem. 1995, 46, 305-334.

(33) Flach, C. R.; Gericke, A.; Mendelsohn, R. J. Phys. Chem. B 1997, $101,58-65$.

(34) Andreeva, T. D.; Petrov, J. G.; Brezesinski, G.; Möhwald, H. Langmuir 2008, 24, 8001-8007.

(35) Lepère, M.; Muenter, A. H.; Chevallard, C.; Guenoun, P.; Brezesinski, G. Colloids Surf. A 2007, 303, 73-78.

(36) Muenter, A. H.; Hentschel, J.; Börner, H. G.; Brezesinski, G. Langmuir 2008, 24, 3306-3316.

(37) Fainerman, V. B.; Vollhardt, D. J. Phys. Chem. B 1999, 103, 145150 .

(38) Bringezu, F.; Dobner, B.; Brezesinski, G. Chem.-Eur. J. 2002, 8, 3203-3210.

(39) Bellamy, L. J. The Infrared Spectra of Complex Molecules, 2nd ed.; John Wiley \& Sons: New York, 1958; Chapter 12; pp 203-233.

(40) Smith, L. A. In Applied Infrared Spectroscopy. Fundamentals, Techniques and Analytical Problem Solving; Elving, P. J., Winefordner, J. D., Eds.; John Wiley \& Sons: New York/Chichester/Brisbane/Toronto, 1979; Vol. 54, Appendix 2; p 290.

(41) Rusanov, A. I. Fazovye Ravnovesija i Poverchnostnye Javlenija; Khimija: Leningrad, 1967.

JP104358Z 